\title{
Vascular injuries in the state of Pará, Brazil, 2011-2013 and their relation with demographic and clinical variables
}

\author{
Traumatismos vasculares no Estado do Pará, Brasil, período de 2011 a 2013, e sua \\ relação com variáveis clínicas e demográficas
}

Ludmylla Teixeira Soares ${ }^{1}$, Carina Corrêa Bastos ${ }^{2}$, Adib Koury Junior ${ }^{3}$, Aldo José Fontes Pereira ${ }^{4}$

\begin{abstract}
Background: Vascular traumas are associated with high morbidity rates. Objective: To report the characteristics of vascular traumas in the Brazilian state of Pará, in trauma victims treated at the Hospital Metropolitano de Urgência e Emergência (HMUE), from 2011 to 2013. Method: This was a descriptive, cross-sectional, retrospective and quantitative study that analyzed data on sex, age group, geographical origin, time waiting for care, mechanism of trauma, clinical status, anatomic site of injury, prevalence of associated fractures, vascular structures injured, types of vascular injury, principal types of surgery, early postoperative outcomes, level of amputation, number of deaths, length of hospital stay and multidisciplinary care for 264 medical records. Results: The majority of victims were male and the most common age group was from 16 to 30 years. The majority of cases were from towns other than the state capital, accounting for 169 cases (64.02\%). The principal mechanism of injury was firearm wounding - $110(41.67 \%)$ followed by cold weapon wounds $-65(24.62 \%)$ and traffic accidents - $42(15.91 \%)$. The segments of the body and the vascular structures most often injured were lower limbs - 120 (45.45\%) and injuries to the popliteal and femoral arteries and veins. The most common clinical presentation at admission was hemorrhage - 154 (58.33\%). The most common surgeries were ligatures of veins and arteries. There were $163(61.74 \%)$ hospital discharges and $33(12.5 \%)$ deaths. Conclusions: The greatest prevalence observed was related to traumas caused by urban violence. Victims were most frequently male, of working age and from towns other than the capital of the state of Pará.
\end{abstract}

Keywords: trauma; injuries of the vascular system; vascular surgical procedures; amputation.

\begin{abstract}
Resumo
Contexto: Os traumatismos vasculares estão relacionados a altas taxas de morbidade. Objetivo: Caracterizar o traumatismo vascular no Estado do Pará, em vítimas atendidas no Hospital Metropolitano de Urgência e Emergência (HMUE), no período de 2011 a 2013. Método: Trata-se de um estudo descritivo, transversal, retrospectivo, quantitativo, que analisou: sexo, faixa etária, procedência, tempo de espera pelo atendimento, mecanismo de trauma, quadro clínico, região anatômica acometida, prevalência de fraturas associadas, estrutura vascular, tipos de lesão vascular, principais tipos de cirurgias, evolução do pós-operatório, nível de amputação, número de óbitos, tempo de internação e atendimento multiprofissional, dentre 264 prontuários. Resultados: A maioria das vítimas foi do sexo masculino e a faixa etária mais acometida foi entre 16 a 30 anos. A maioria dos casos foi procedente de fora da capital, perfazendo 169 casos (64,02\%). O principal mecanismo de lesão foi por arma de fogo - 110 (41,67\%), seguido por arma branca - 65 (24,62\%) e acidente de trânsito - 42 (15,91\%). O segmento corporal e as estruturas vasculares mais acometidas foram os membros inferiores - 120 (45,45\%), com lesões de artéria e veia poplítea e femoral. Durante a admissão, 0 quadro clínico mais frequente foi a hemorragia - 154 (58,33\%). As ligaduras de veias e artérias foram as cirurgias mais frequentes. Houve $163(61,74 \%)$ altas e 33 (12,5\%) óbitos. Conclusão: A maior prevalência encontrada foi referente a traumas decorrentes da violência urbana, sendo também frequente o acometimento de indivíduos do sexo masculino, em idade produtiva e não procedentes da capital do Estado do Pará.
\end{abstract}

Palavras-chave: trauma; lesões do sistema vascular; procedimentos cirúrgicos vasculares; amputação.

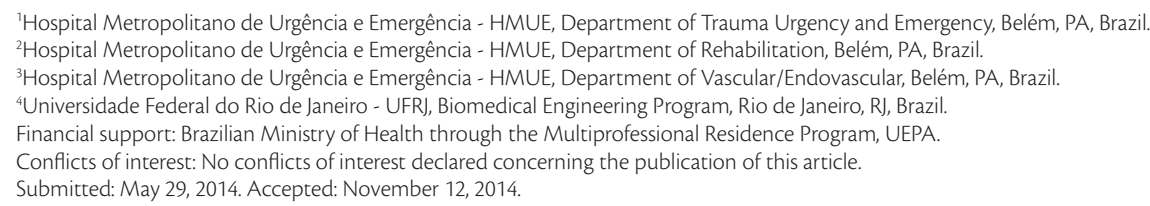




\section{INTRODUCTION}

Traumatisms can be considered a modern pandemic and one of the greatest current challenges, with rates that have both economic and psychosocial impacts on society and victims who may be young people or adults, civilians or military personnel. ${ }^{1,2}$ Vascular injuries traditionally predominated among military populations, caused by wars. ${ }^{3}$ In 2011 , a progressive increase in the number of cases was reported, but with a predominance of male, civilian victims of working age. ${ }^{2,4}$

According to the World Health Organization (WHO), ${ }^{5}$ vascular traumas account for $8 \%$ of traumas, and severe vascular injury incidence is related to high mortality and morbidity rates. ${ }^{1,4,6}$ More specifically, vascular traumas are generally related to severe traumatic injuries, such as fractures and amputations, ${ }^{4}$ resulting in increased surgical postoperative complications, morbidity and incapacity in victims. ${ }^{3,4}$ This scenario has been found to be common in Brazilian hospitals providing urgent and emergency care in urban centers. ${ }^{7}$

Modern agents of trauma, such as vehicles at high speed, modern weapons, highly inflammable materials and others, transmit high energy when they impact the human body and are capable of causing complex injuries to tissues, ${ }^{1,2,8}$ and injuries to the head and brain and the blood vessels are the most common causes of mortality and morbidity. ${ }^{7}$ As society modernizes, advances are also achieved in surgery and in techniques for rehabilitation, all over the world. ${ }^{8}$ However, few initiatives have had a significant impact in promoting awareness and prevention of the many types of accidents ${ }^{9}$ and acts of violence.

It is known that planning of preventative healthcare interventions is dependent on studies based on scientific evidence, ${ }^{9}$ providing data on the incidence and/or prevalence of cases; ${ }^{10}$ such as epidemiological studies for example. In view of the current scarcity of studies investigating the subject in general ${ }^{11}$ and specifically of the absence of evidence-based data on vascular traumas in the Brazilian state of Pará, this study was conducted with the objective of reporting the characteristics of the profile of vascular traumatisms in the state during the period from February 2011 to March 2013, by studying trauma victims seen at a trauma referral center, the Hospital Metropolitano de Urgência e Emergência (HMUE).

\section{METHODS}

This is a descriptive, cross-sectional, observational and retrospective epidemiological study based on data collected from medical records. It was conducted in accordance with applicable guidelines on research involving human beings, as set out in Brazilian National Health Council (Conselho Nacional de Saúde) resolution CNS 466/12, and was approved by the Research Ethics Committee at the Universidade do Estado do Pará biological and health sciences department (Plataforma Brasil reference number 279.942).

The study was conducted at the HMUE, which is the only trauma referral center in the state of Pará, and the data analyzed were provided by the hospital's statistics department. Data were acquired from all medical records for patients who had been victims of vascular injuries and had been admitted to the HMUE from February 2011 to March 2013 and identified by the following International Classification of Diseases (ICD) codes or their subcodes: 10.S25.1; 10.S35; 10.S35.1; 10.S35.4; 10.S45.0; 10.S45.1; 10.S45.2; 10.S55.0; 10.S65.1; 10.S75.0; 10.S75.1; 10.S85.0; 10.S85.1; 10.S85.5; 10.S95. Operations conducted by the hospital's vascular surgeons were also reviewed. Medical records were excluded if they did not contain a record of vascular surgery identified by an ICD code, if they did not mention the hospital's vascular surgeons or if the victims were not residents of the state of Pará.

\section{Data collection}

Data were collected on the following variables: sex, age group, place of origin, means of transport employed for journey to hospital, interval of time between suffering trauma and being seen at hospital, mechanism of trauma, anatomic site of injury, vascular structure injured, clinical presentation, types of surgery, early postoperative outcomes, amputations, length of hospital stay and postoperative care provided by members of the rehabilitation service (by specialty) to patients treated at the only trauma referral hospital in the state of Pará.

\section{Statistical analysis}

A descriptive analysis of the data was conducted and the Chi-square test with Yates' correction factor was used to test for associations between categorical variables. When the Chi-square test of independence was employed, Karl Pearson's contingency coefficient (CC) was calculated to indicate the degree of association between variables. Variables were grouped in order to make the sample more homogenous and appropriate for the statistical tests. The Mann-Whitney test was used to test for differences between ages of male and female victims. For all tests the significance level was set at $\alpha=0.05$, considering a $95 \%$ confidence 
interval. Statistical analyses were conducted using SPSS software (version 20.0, IBM Corporation, New York, United States).

\section{RESULTS}

After review of 292 medical records, 264 records were selected for analysis. Tables 1, 2 and 3 list the characteristics of the vascular trauma victims in the resulting sample.

From this total, $251(95.08 \%)$ patients were male and $13(04.92 \%)$ were female $\left[\chi^{2}(1)=214.56 ; p<0.0001\right]$, although the mean of ages of male $(28.98 \pm 12.33)$ and female victims $(34.85 \pm 24.23)$ were similar and there was no statistically significant difference $[\mathrm{U}=1541.5 ; 95 \% \mathrm{CI}=-6.00$ to $10.00 ; \mathrm{p}=0.74]$. The victims' geographical origins broke down as follows: $95(35.98 \%)$ were from the state capital Belém and 169 $(64.02 \%)$ were from other towns in the state of Pará $\left[\chi^{2}(1)=20.74 ; p<0.0001\right]$. The total number of deaths was $33(12.5 \%)$, just five of which occurred during surgery. Table 4 shows the frequency distribution of principal injuries to arteries and veins.

There was a statistically significant association between mechanism of trauma and anatomic site of injury $[\chi 2(6)=50.25 ; C C=0.4 ; p \leq 0.001]$ (Table 5).
There were also associations between the structure that suffered injury and clinical presentation of vascular trauma cases $\left[\chi^{2}(6)=21.61 ; C C=0.3 ; p=0.001\right]$ (Table 6 ), and between description of vascular injury and structure injured $\left[\chi^{2}(6)=66.68 ; \mathrm{CC}=0.4 ; \mathrm{p}<0.001\right]$ (Table 7). There was no association between time before patient admitted to hospital (more than 8 hours, 3-6 hours, or less than 3 hours) and amputation rates $\left[\chi^{2}(2)=0.58 ; \mathrm{CC}=0.05 ; \mathrm{p}=0.58\right]$.

It was also possible to trace a relationship between type of surgery and outcomes during the postoperative period, with ligature surgery most frequently preceding both discharge and death of patients (Table 8).

\section{DISCUSSION}

Analyses of the characteristics of vascular traumas are normally based on data from military casualties during times of war. In view of the current scarcity of studies investigating the characteristics of vascular traumatisms in the civilian population and the absence of data illustrating the profile of vascular trauma victims in the Brazilian state of Pará, this study has characterized for the first time vascular traumas in the state and related them to clinical and demographic

Table 1. Characteristics of 264 vascular traumatism victims treated at the Hospital Metropolitano de Urgência e Emergência: age group, mechanism of trauma, time before receiving hospital care, transportation and amputation level - Pará, Brazil, 2011-2013, absolute totals (frequencies) and percentages in parentheses.

\begin{tabular}{|c|c|c|c|}
\hline Age group (years) ${ }^{\mathrm{a}}$ & $(n=264)$ & $\begin{array}{c}\text { Time before receiving } \\
\text { hospital care }\end{array}$ & $(n=264)$ \\
\hline$<15$ & $16(6.06)$ & $<1$ hour & $2(0.75)$ \\
\hline $16-30$ & $154(58.33)$ & $1-3$ hours & $23(8.71)$ \\
\hline $31-46$ & $71(26.89)$ & 3-6 hours & $92(34.85)$ \\
\hline $47-62$ & $16(6.06)$ & 6-8 hours & $3(1.14)$ \\
\hline $63-78$ & $6(2.27)$ & $>8$ hours & $144(54.55)$ \\
\hline$>79$ & $1(0.38)$ & & \\
\hline Mechanism of traumab & $(n=264)$ & Transportation $^{\mathrm{d}}$ & $(n=264)$ \\
\hline Firearm & $110(41.67)$ & SAMU emergency ambulance service & $210(79.55)$ \\
\hline Cold weapon $^{\dagger}$ & $65(24.62)$ & Private transport & $36(13.64)$ \\
\hline Traffic & $42(15.91)$ & Fire service & $10(3.79)$ \\
\hline Workplace accident & $13(04.92)$ & Police patrol car & $8(3.03)$ \\
\hline Blunt trauma & $11(04.17)$ & Level of amputation $^{e}$ & $(n=53)$ \\
\hline Penetrating trauma ${ }^{\#}$ & $6(02.27)$ & Finger & $4(07.69)$ \\
\hline Fall & $6(02.27)$ & Hand & $1(01.92)$ \\
\hline Burn & $5(01.89)$ & Forearm & $6(11.54)$ \\
\hline Surgical procedure & $4(01.52)$ & Arm & $4(07.69)$ \\
\hline Animal bite & $1(0.38)$ & Thigh & $27(51.93)$ \\
\hline \multirow[t]{3}{*}{ Snake bite } & $1(0.38)$ & Leg & $7(13.46)$ \\
\hline & & Foot & $2(03.77)$ \\
\hline & & Toe & 1 (01.92) \\
\hline
\end{tabular}

\#: accidents with wood, glass and foreign objects. $\dagger$ : blades. a: $\chi^{2}(5)=402.05, p<0.0001$. b: $\chi^{2}(10)=506.58, p<0.0001$. c: $\chi^{2}(4)=299.30, p<0.0001$. d: $\chi^{2}(3)=426.31$, p $<0.0001$. e: $\chi^{2}(7)=79.08, p<0.0001$. 
Table 2. Characteristics of 264 vascular traumatism victims treated at the Hospital Metropolitano de Urgência e Emergência: site of injury, description of vascular injury, type of surgery, structure injured, clinical presentation and postoperative outcomes - Pará, Brazil, 2011-2013, absolute totals (frequencies) and percentages in parentheses.

\begin{tabular}{|c|c|c|c|}
\hline Site of injury ${ }^{\mathrm{a}}$ & $(n=264)$ & Structure injured ${ }^{d}$ & $(n=264)$ \\
\hline Lower limbs & $120(45.45)$ & Artery & $102(38.64)$ \\
\hline Upper limbs & $93(35.23)$ & Vein & $18(6.82)$ \\
\hline Thorax & $19(7.20)$ & Artery/Vein & $72(27.27)$ \\
\hline Neck & $16(6.06)$ & Heart & $3(1.14)$ \\
\hline Abdomen & $13(4.92)$ & Muscular Vessels & $66(25.00)$ \\
\hline Face & $1(0.38)$ & Mesenteric Vessels & $3(1.14)$ \\
\hline Pelvis & $1(0.38)$ & & \\
\hline Penis & $1(0.38)$ & & \\
\hline Description of vascular injury & $(n=264)$ & Clinical presentation $^{e}$ & $(n=264)$ \\
\hline Partial section & $125(47.35)$ & Active hemorrhage & $154(58.33)$ \\
\hline Total section & $51(19.32)$ & Hematoma & $48(18.18)$ \\
\hline Thrombosis & $8(3.03)$ & Ischemia & $24(9.09)$ \\
\hline Arteriovenous fistula & $1(0.38)$ & Hypovolemic shock & $20(7.58)$ \\
\hline \multirow[t]{2}{*}{ Others (NID) } & $79(29.92)$ & Compartment syndrome & $16(6.06)$ \\
\hline & & Cardiac tamponade syndrome & $2(0.76)$ \\
\hline Types of surgeryc & $(n=264)$ & Early postoperative outcomes ${ }^{f}$ & $(n=264)$ \\
\hline Ligature & $80(30.30)$ & Discharge & $163(61.74)$ \\
\hline Vascular exploration & $43(16.29)$ & Amputation & $37(14.02)$ \\
\hline Autologous graft & $39(14.77)$ & Death & $28(10.61)$ \\
\hline Amputation & $33(12.50)$ & Infection & $25(9.47)$ \\
\hline Anastomosis & $17(6.44)$ & Compartment syndrome & $2(0.76)$ \\
\hline Fasciotomy & $16(6.06)$ & Functional limitation & $3(1.14)$ \\
\hline Revascularization & $15(5.68)$ & Pseudoaneurysm & $1(0.38)$ \\
\hline Pseudoaneurysm repair & $12(4.55)$ & Dehiscence & $1(0.38)$ \\
\hline Suture & $5(1.89)$ & Fasciotomy & $4(1.52)$ \\
\hline Pericardical window & $2(0.76)$ & & \\
\hline Synthetic graft & $1(0.38)$ & & \\
\hline
\end{tabular}

NID: the type of vascular injury was not described on the medical record. a: $\chi^{2}(7)=458.36, p<0.0001 . b: \chi^{2}(4)=200.62, p<0.0001$. c: $\chi^{2}(10)=230.66, p<0.0001$. d: $\chi^{2}(5)=197.05, p<0.0001$. e: $\chi^{2}(5)=355.46, p<0.0001 . f: \chi^{2}(8)=737.53, p<0.0001$.

Table 3. Characteristics of 264 vascular traumatism victims treated at the Hospital Metropolitano de Urgência e Emergência: presence of fractures, type of surgery, readmission, length of hospital stay and rehabilitation professionals providing care - Pará, Brazil, 2011-2013, absolute totals (frequencies) and percentages in parentheses.

\begin{tabular}{|c|c|c|c|c|c|}
\hline Fractures $^{\mathrm{a}}$ & $(n=264)$ & Surgery $^{d}$ & $(n=264)$ & Readmission ${ }^{i}$ & $(n=264)$ \\
\hline Yes & $188(71.21)$ & Immediate & $178(67.42)$ & Yes & $55(20.83)$ \\
\hline No & $76(28.79)$ & Not immediate & $86(32.58)$ & No & $209(79.17)$ \\
\hline Length of stay & $\begin{array}{l}\text { Hospital } \\
(\mathrm{n}=264)^{\mathrm{b}}\end{array}$ & $\operatorname{ICU}(n=44)^{c}$ & Rehabilitation professionals & Yes & No \\
\hline$<1$ week & $150(56.82)$ & $32(72.73)$ & Physiotherapist $^{e}$ & $98(37.12)$ & $166(62.88)$ \\
\hline 2-4 weeks & $74(28.03)$ & $7(15.91)$ & Psychologist $^{f}$ & $30(11.36)$ & $234(88.64)$ \\
\hline $1-2$ months & $32(12.12)$ & $5(11.36)$ & Occupational therapist ${ }^{g}$ & $14(05.30)$ & $250(94.70)$ \\
\hline $2-3$ months & $3(01.14)$ & - & Speech and hearing therapist ${ }^{\mathrm{h}}$ & $3(01.14)$ & $261(98.86)$ \\
\hline$>3$ months & $5(01.89)$ & - & & & \\
\hline
\end{tabular}

a: $\chi^{2}(1)=47.52, p<0.0001$. b: $\chi^{2}(4)=285.89, p<0.0001$. c: $\chi^{2}(2)=30.86, p<0.0001$. d: $\chi^{2}(1)=32.06, p<0.0001$. e: $\chi^{2}(1)=17.52, p<0.0001 . f: \chi^{2}(1)=157.64, p<0.0001$. g: $\chi^{2}(1)=210.97, p<0.0001$. h: $\chi^{2}(1)=252.14, p<0.0001$. i: $\chi^{2}(1)=89.84, p<0.0001$. 
Table 4. Frequencies of involvement of arteries and veins injured by vascular traumas among cases treated at the Hospital Metropolitano de Urgência e Emergência - Pará, Brazil, 2011-2013, absolute totals (frequencies) and percentages in parentheses.

\begin{tabular}{|c|c|c|c|}
\hline Arteries involved $^{+}$ & $(n=264)$ & Veins involved $^{\#}$ & $(n=264)$ \\
\hline Common carotid & $4(1.52)$ & Internal jugular & $1(0.38)$ \\
\hline External carotid & $3(1.14)$ & External jugular & $4(1.52)$ \\
\hline Subclavian & $9(3.41)$ & Subclavian & $4(1.52)$ \\
\hline Axillary & $9(3.41)$ & Superior vena cava & $2(0.76)$ \\
\hline Brachial & $26(9.85)$ & Abdominal inferior vena cava & $3(1.14)$ \\
\hline Radial & $7(2.65)$ & Axillary & $3(1.14)$ \\
\hline Ulnar & $18(6.82)$ & Brachial & $8(3.03)$ \\
\hline Radial and ulnar & $6(2.27)$ & Basilic & $5(1.89)$ \\
\hline Abdominal aorta & $2(0.76)$ & Ulnar & $2(0.76)$ \\
\hline Mesenteric & $7(2.65)$ & Common iliac & $4(1.52)$ \\
\hline Common iliac & $1(0.38)$ & Common femoral & $9(3.41)$ \\
\hline Internal iliac & $1(0.38)$ & Deep femoral & $2(0.76)$ \\
\hline Common femoral & $9(3.41)$ & Superficial femoral & $13(4.92)$ \\
\hline Superficial femoral & $15(5.68)$ & Great saphenous & $5(1.89)$ \\
\hline Deep femoral & $2(0.76)$ & Popliteal & $11(4.17)$ \\
\hline Popliteal & $19(7.20)$ & Tibial & $5(1.89)$ \\
\hline Anterior tibial & $7(2.65)$ & Deep veins of lower limbs & $114(43.18)$ \\
\hline Posterior tibial & $8(3.03)$ & No venous injuries & $69(26.14)$ \\
\hline Anterior and posterior tibials & $3(1.14)$ & - & - \\
\hline Fibular & $1(0.38)$ & - & - \\
\hline Plantar & $1(0.38)$ & - & - \\
\hline Muscular vessels & $100(37.88)$ & - & - \\
\hline No arterial injuries & $6(2.27)$ & _ & _- \\
\hline
\end{tabular}

Table 5. Associations between mechanism of trauma and anatomic site of injury in cases treated at the Hospital Metropolitano de Urgência e Emergência - Pará, Brazil, 2011-2013.

\begin{tabular}{|c|c|c|c|c|c|c|c|}
\hline \multirow{2}{*}{ Mechanism of trauma } & \multicolumn{6}{|c|}{ Anatomic Site } & \multirow{2}{*}{ Total n (\%) } \\
\hline & \multicolumn{2}{|c|}{ Lower Limbs } & \multicolumn{2}{|c|}{ Upper Limbs } & \multicolumn{2}{|c|}{ FNTAP $^{+}$} & \\
\hline \multirow[t]{2}{*}{ Wound by cold weapon } & \multirow{2}{*}{15} & $23.08^{a}$ & \multirow{2}{*}{36} & $55.38^{\mathrm{a}}$ & \multirow{2}{*}{14} & $21.54^{\mathrm{a}}$ & \multirow{2}{*}{$65(24.62)$} \\
\hline & & $12.50^{\mathrm{b}}$ & & $38.71^{\mathrm{b}}$ & & $27.45^{\mathrm{b}}$ & \\
\hline \multirow[t]{2}{*}{ Firearm wound } & \multirow{2}{*}{60} & $54.55^{\mathrm{a}}$ & \multirow{2}{*}{24} & $21.82^{\mathrm{a}}$ & \multirow{2}{*}{26} & $23.64^{\mathrm{a}}$ & \multirow{2}{*}{$110(41.67)$} \\
\hline & & $50.00^{\mathrm{b}}$ & & $25.81^{\mathrm{b}}$ & & $50.98^{\mathrm{b}}$ & \\
\hline \multirow[t]{2}{*}{ Traffic accident } & \multirow{2}{*}{31} & $73.81^{\mathrm{a}}$ & \multirow{2}{*}{5} & $11.90^{\mathrm{a}}$ & \multirow{2}{*}{6} & $14.29^{\mathrm{a}}$ & \multirow{2}{*}{$42(15.91)$} \\
\hline & & $25.83^{\mathrm{b}}$ & & $05.38^{\mathrm{b}}$ & & $11.76^{\mathrm{b}}$ & \\
\hline \multirow[t]{2}{*}{ Others ${ }^{*}$} & \multirow{2}{*}{14} & $29.79^{a}$ & \multirow{2}{*}{28} & $59.57^{\mathrm{a}}$ & \multirow{2}{*}{5} & $10.64^{\mathrm{a}}$ & \multirow{2}{*}{$47(17.80)$} \\
\hline & & $11.67^{\mathrm{b}}$ & & $30.11^{\mathrm{b}}$ & & $09.80^{\mathrm{b}}$ & \\
\hline Total n (\%) & $120(45.45)$ & & & & $51(19.32)$ & & $264(100.00)$ \\
\hline
\end{tabular}

variables, thereby correcting the lack of a profile of the victims of these traumas in the state of Pará.

A total of 264 medical records were selected for study, relating to trauma victims from the state of Pará treated at the HMUE from February 2011 to March 2013. This time frame was chosen because it corresponds to an initiative by the state of Pará to make the HMUE a center of excellence for urgent and emergency treatment, providing 24-hour care for traumas of all levels of complexity, with the result that treatment for vascular traumas became the most common type of care provided at this hospital. The number of medical records analyzed was considerable, ${ }^{4,12,13}$ enabling analysis of $90.41 \%$ of all vascular traumas treated at the HMUE during the period analyzed. 
Table 6. Associations between structure injured and clinical presentation in cases treated at the Hospital Metropolitano de Urgência e Emergência - Pará, Brazil, 2011-2013.

\begin{tabular}{|c|c|c|c|c|c|c|c|}
\hline \multirow{2}{*}{ Structure injured } & \multicolumn{6}{|c|}{ Clinical presentation } & \multirow{2}{*}{ Total n (\%) } \\
\hline & \multicolumn{2}{|c|}{ Active hemorrhage } & \multicolumn{2}{|c|}{ Hematoma } & \multicolumn{2}{|c|}{ Others $^{\dagger}$} & \\
\hline \multirow[t]{2}{*}{ Artery } & \multirow[b]{2}{*}{67} & $65.69^{\mathrm{a}}$ & \multirow[b]{2}{*}{25} & $24.51^{\mathrm{a}}$ & \multirow{2}{*}{10} & $9.81^{\mathrm{a}}$ & \multirow{2}{*}{$102(38.64)$} \\
\hline & & $43.51^{b}$ & & $52.08^{b}$ & & $16.13^{\mathrm{b}}$ & \\
\hline \multirow[t]{2}{*}{ Artery/ vein } & \multirow{2}{*}{38} & $52.78^{\mathrm{a}}$ & \multirow{2}{*}{8} & $11.11^{\mathrm{a}}$ & \multirow{2}{*}{26} & $36.11^{\mathrm{a}}$ & \multirow{2}{*}{$72(27.27)$} \\
\hline & & $24.68^{b}$ & & $16.67^{\mathrm{b}}$ & & $41.94^{\mathrm{b}}$ & \\
\hline \multirow[t]{2}{*}{ Muscular vessels } & \multirow{2}{*}{37} & $56.06^{\mathrm{a}}$ & \multirow{2}{*}{9} & $13.64^{\mathrm{a}}$ & \multirow{2}{*}{20} & $30.30^{\mathrm{a}}$ & \multirow{2}{*}{$66(25.00)$} \\
\hline & & $24.06^{\mathrm{b}}$ & & $18.75^{b}$ & & $32.26^{\mathrm{b}}$ & \\
\hline \multirow[t]{2}{*}{ Others" } & \multirow{2}{*}{12} & $50.00^{\mathrm{a}}$ & \multirow{2}{*}{6} & $25.00^{\mathrm{a}}$ & \multirow{2}{*}{6} & $25.00^{\mathrm{a}}$ & \multirow{2}{*}{$24(9.09)$} \\
\hline & & $7.79^{\mathrm{b}}$ & & $12.50^{\mathrm{b}}$ & & $9.68^{\mathrm{b}}$ & \\
\hline Total n (\%) & \multicolumn{2}{|c|}{$154(58.33)$} & \multicolumn{2}{|c|}{$48(18.18)$} & \multicolumn{2}{|c|}{$62(23.49)$} & $264(100.00)$ \\
\hline
\end{tabular}

\#: ischemia, hypovolemic shock, compartment syndrome and cardiac tamponade syndrome. $\dagger$ : vein, heart and mesenteric vessels. a: percentage in relation to structure injured. b: percentage in relation to clinical presentation. $\chi^{2}(6)=21.61 ; C C=0.3 ; p=0.001$.

Table 7. Associations between description of vascular injury and structure injured in cases treated at the Hospital Metropolitano de Urgência e Emergência - Pará, Brazil, 2011-2013.

\begin{tabular}{|c|c|c|c|c|c|c|c|c|c|}
\hline \multirow{2}{*}{$\begin{array}{l}\text { Description of } \\
\text { vascular injury }\end{array}$} & \multicolumn{9}{|c|}{ Structure injured } \\
\hline & \multicolumn{2}{|c|}{ Artery } & \multicolumn{2}{|c|}{ Artery/ vein } & \multicolumn{2}{|c|}{ Muscular vessels } & \multicolumn{2}{|c|}{ Others $^{\dagger}$} & Total n (\%) \\
\hline \multirow[t]{2}{*}{ Partial section } & \multirow{2}{*}{67} & $53.60^{a}$ & \multirow{2}{*}{35} & $28.00^{\mathrm{a}}$ & \multirow{2}{*}{11} & $8.80^{\mathrm{a}}$ & \multirow{2}{*}{12} & $9.60^{\mathrm{a}}$ & \multirow{2}{*}{$125(47.35)$} \\
\hline & & $65.69^{\mathrm{b}}$ & & $48.61^{b}$ & & $16.66^{\mathrm{b}}$ & & $50.00^{\mathrm{b}}$ & \\
\hline \multirow[t]{2}{*}{ Total section } & \multirow{2}{*}{17} & $33.33^{a}$ & \multirow{2}{*}{22} & $43.14^{\mathrm{a}}$ & \multirow{2}{*}{8} & $15.69^{\mathrm{a}}$ & \multirow{2}{*}{4} & $7.84^{\mathrm{a}}$ & \multirow{2}{*}{$51(19.32)$} \\
\hline & & $16.67^{\mathrm{b}}$ & & $30.56^{\mathrm{b}}$ & & $12.12^{\mathrm{b}}$ & & $16.67^{b}$ & \\
\hline \multirow[t]{2}{*}{ Others ${ }^{\#}$} & \multirow{2}{*}{18} & $20.46^{\mathrm{a}}$ & \multirow{2}{*}{15} & $17.05^{\mathrm{a}}$ & \multirow{2}{*}{47} & $53.41^{\mathrm{a}}$ & \multirow{2}{*}{8} & $9.09^{\mathrm{a}}$ & \multirow{2}{*}{$88(33.33)$} \\
\hline & & $17.65^{b}$ & & $20.83^{b}$ & & $71.21^{b}$ & & $33.33^{\mathrm{b}}$ & \\
\hline Total n (\%) & \multicolumn{2}{|c|}{$102(38.64)$} & \multicolumn{2}{|c|}{$72(27.27)$} & \multicolumn{2}{|c|}{$66(25.00)$} & \multicolumn{2}{|c|}{$24(9.09)$} & $\begin{array}{c}264 \\
(100.00)\end{array}$ \\
\hline
\end{tabular}

\#: thrombosis, arteriovenous fistula and others. t: vein, heart and mesenteric vessels. a: percentage in relation to description of vascular injury; b: percentage in relation to structure injured. $\chi^{2}(6)=66.68 ; C C=0.4 ; p<0.001$.

Table 8. Relationship between type of surgery and early postoperative outcomes in cases treated at the Hospital Metropolitano de Urgência e Emergência - Pará, Brazil, 2011-2013.

\begin{tabular}{|c|c|c|c|c|c|c|c|c|c|c|c|}
\hline \multirow{4}{*}{$\begin{array}{l}\text { Types de surgery } \\
\text { Amputation }\end{array}$} & \multicolumn{11}{|c|}{ Early postoperative outcomes } \\
\hline & \multicolumn{2}{|c|}{ Discharge } & \multicolumn{2}{|c|}{ Amputation } & \multicolumn{2}{|c|}{ Infection } & \multicolumn{2}{|c|}{ Death } & \multicolumn{2}{|c|}{ Others $^{\dagger}$} & \multirow{3}{*}{$\begin{array}{c}\begin{array}{c}\text { Total } \mathrm{n} \\
(\%)\end{array} \\
33 \\
(12.50)\end{array}$} \\
\hline & \multirow{2}{*}{2} & $6.06^{\mathrm{a}}$ & \multirow{2}{*}{24} & $72.73^{\mathrm{a}}$ & \multirow{2}{*}{6} & $18.18^{\mathrm{a}}$ & \multirow{2}{*}{1} & $3.03^{\mathrm{a}}$ & \multirow{2}{*}{0} & $0.00^{\mathrm{a}}$ & \\
\hline & & $1.23^{\mathrm{b}}$ & & $64.87^{\mathrm{b}}$ & & $24.00^{b}$ & & $3.57^{\mathrm{b}}$ & & $0.00^{\mathrm{b}}$ & \\
\hline \multirow[t]{2}{*}{ Pseudoaneurysm repair } & \multirow{2}{*}{8} & $66.67^{\mathrm{a}}$ & \multirow{2}{*}{0} & $0.00^{\mathrm{a}}$ & \multirow{2}{*}{2} & $16.67^{\mathrm{a}}$ & \multirow{2}{*}{1} & $8.33^{\mathrm{a}}$ & \multirow{2}{*}{1} & $8.33^{\mathrm{a}}$ & \multirow{2}{*}{$\begin{array}{c}12 \\
(4.55)\end{array}$} \\
\hline & & $4.91^{b}$ & & $0.00^{\mathrm{b}}$ & & $8.00^{\mathrm{b}}$ & & $3.57^{b}$ & & $9.09^{b}$ & \\
\hline \multirow[t]{2}{*}{ Autologous graft } & \multirow{2}{*}{30} & $76.92^{\mathrm{a}}$ & \multirow{2}{*}{2} & $5.13^{\mathrm{a}}$ & \multirow{2}{*}{2} & $5.13^{\mathrm{a}}$ & \multirow{2}{*}{2} & $5.13^{\mathrm{a}}$ & \multirow{2}{*}{3} & $7.69^{\mathrm{a}}$ & 39 \\
\hline & & $18.41^{\mathrm{b}}$ & & $5.41^{\mathrm{b}}$ & & $8.00^{\mathrm{b}}$ & & $7.14^{\mathrm{b}}$ & & $27.27^{b}$ & $(14.77)$ \\
\hline \multirow[t]{2}{*}{ Vascular exploration } & \multirow{2}{*}{30} & $69.77^{\mathrm{a}}$ & \multirow{2}{*}{0} & $0.00^{\mathrm{a}}$ & \multirow{2}{*}{3} & $6.98^{\mathrm{a}}$ & \multirow{2}{*}{8} & $18.61^{\mathrm{a}}$ & \multirow{2}{*}{2} & $4.65^{\mathrm{a}}$ & 43 \\
\hline & & $18.41^{\mathrm{b}}$ & & $0.00^{\mathrm{b}}$ & & $12.00^{\mathrm{b}}$ & & $28.57^{b}$ & & $18.18^{b}$ & $(16.28)$ \\
\hline \multirow[t]{2}{*}{ Fasciotomy } & & $62.50^{\mathrm{a}}$ & & $12.50^{\mathrm{a}}$ & & $18.75^{\mathrm{a}}$ & & $0.00^{\mathrm{a}}$ & & $6.25^{\mathrm{a}}$ & 16 \\
\hline & 10 & $6.13^{b}$ & 2 & $5.40^{\mathrm{b}}$ & 3 & $12.00^{\mathrm{b}}$ & 0 & $0.00^{\mathrm{b}}$ & 1 & $9.09^{\mathrm{b}}$ & $(6.06)$ \\
\hline Ligature & & $65.00^{\mathrm{a}}$ & & $7.50^{\mathrm{a}}$ & & $8.75^{\mathrm{a}}$ & & $16.25^{\mathrm{a}}$ & & $2.50^{\mathrm{a}}$ & 80 \\
\hline & 52 & $31.90^{\mathrm{b}}$ & 6 & $16.22^{\mathrm{b}}$ & 7 & $28.00^{\mathrm{b}}$ & 13 & $46.43^{b}$ & 2 & $18.18^{\mathrm{b}}$ & $(30.30)$ \\
\hline Anastomosis & & $70.59^{\mathrm{a}}$ & & $11.76^{a}$ & & $5.88^{\mathrm{a}}$ & & $11.76^{a}$ & & $0.00^{\mathrm{a}}$ & 17 \\
\hline & 12 & $7.36^{\mathrm{b}}$ & 2 & $5.40^{\mathrm{b}}$ & 1 & $4.00^{\mathrm{b}}$ & 2 & $7.14^{b}$ & 0 & $0.00^{\mathrm{b}}$ & $(6.44)$ \\
\hline Revascularization & 12 & $80.00^{\mathrm{a}}$ & 1 & $6.67^{\mathrm{a}}$ & 1 & $6.67^{\mathrm{a}}$ & 0 & $0.00^{\mathrm{a}}$ & 1 & $6.67^{\mathrm{a}}$ & 15 \\
\hline & 12 & $7.36^{\mathrm{b}}$ & 1 & $2.70^{\mathrm{b}}$ & 1 & $4.00^{\mathrm{b}}$ & 0 & $0.00^{\mathrm{b}}$ & 1 & $9.09^{\mathrm{b}}$ & $(5.68)$ \\
\hline Others" & 7 & $77.78^{\mathrm{a}}$ & & $0.00^{\mathrm{a}}$ & 0 & $0.00^{\mathrm{a}}$ & 1 & $11.11^{\mathrm{a}}$ & 1 & $11.11^{\mathrm{a}}$ & 26 \\
\hline & I & $4.29^{b}$ & 0 & $0.00^{\mathrm{b}}$ & 0 & $0.00^{\mathrm{b}}$ & 1 & $3.57^{b}$ & 1 & $9.09^{\mathrm{b}}$ & $(3.41)$ \\
\hline Total n (\%) & 163 & 1.74) & & (.01) & & 47) & & $0.61)$ & & 17) & $\begin{array}{c}264 \\
(100.00)\end{array}$ \\
\hline
\end{tabular}

\#: suture, pericardical window and synthetic graft. †: compartment syndrome, functional limitation, pseudoaneurysm, dehiscence and fasciotomy. a: percentage in relation to type of surgery. b: percentage in relation to postoperative outcomes. 
In common with other studies, there was a significant predominance of male victims and victims in the age range from 16 to 46 years; ;, $, 3,5,11$ although mean age of patients was similar for both sexes. Of the total sample of 264 trauma victims, $64.02 \%$ were residents of places other than the state capital Belém and the majority were taken to hospital by the SAMU emergency ambulance service, with the result that in the majority of cases there was a considerable interval between the first response and the time at which surgical repair of the injury was attempted. In the majority of cases, this wait was greater than 8 hours. It is probable that the high frequency cases in which transporting the victim took longer than 8 hours is because of the time taken for ambulances to arrive at the place where the trauma occurred and return to the hospital. Shorter times between admission and starting surgery have been related to salvage of injured limbs and their function after vascular repair surgery. ${ }^{3,14}$ However, in the present study there was no association between admission times and amputation cases. This is possibly a result of improved surgical techniques and the quality of care that victims receive on the way to hospital, since, over the last 30 years, trauma centers have been established and an integrated system for care of trauma victims has been implemented, including systematization of initial care and of transportation of trauma victims, making safer removal possible and reducing the mortality rate. ${ }^{14}$

In this study, the total mortality rate was $12.5 \%$ of cases: five patients died during surgical procedures and 28 from complications caused by the traumas. This is a very significant mortality rate, but is in line with the global literature ${ }^{1,4,12,15,16}$ and with a study by Markov et al. ${ }^{17}$ who stated that this type of trauma causes up to $8 \%$ mortality in severe arterial injuries.

In the sample studied here, traumatisms were predominantly caused by firearms, followed by cold weapons and traffic accidents. This is in agreement with the WHO, which lists traffic accidents amongst the causes of vascular traumatisms, since, according to the organization, traffic accidents account for $2.20 \%$ of all deaths in Brazil and the expectation is that by 2030 they will account for $3.60 \%{ }^{5}$ Additionally, in line with the behavioral characteristics of society, vascular injuries have increased in proportion to the number of traffic accidents, attacks with firearms and invasive medical procedures..$^{2,3,11,18}$ Other mechanisms of vascular injury were workplace accidents, blunt traumas, complications of surgical procedures and accidents involving animals or burns. These causes were possibly less frequent because they are more easily prevented. In Brazil, and particularly at urgent and emergency care services, penetrating traumas outweigh blunt traumas and are a significant cause of vascular injuries. ${ }^{2,4,7}$

The findings of this study show that the greatest incidence was of injuries to the lower limbs and the principal vessels injured were the popliteal vein and artery and the superficial femoral vein and artery. It was observed that upper limb injuries were the second most common and the principal vessels injured in upper limbs were the brachial vein and artery. In contrast, data from the Brazilian national health service (SUS) ${ }^{6}$ on the body segments involved in vascular traumas treated during 2004 indicate that in Brazil the incidence of traumatic vascular injuries was greater in upper limbs, at 73.43 per 100,000 admissions, followed by the incidence of traumatic vascular injuries to the lower limbs, at 59.14 per 100,000; showing that there is a difference with relation to the national data. The results of this study possibly do not coincide with the SUS data for the whole of Brazil because the number of traumas caused by firearms in the state of Pará has increased over recent years, in line with an increase in violence in provincial towns. ${ }^{4,19}$ Analysis of the relationship between mechanism of trauma and site of injury confirmed that gunshot wound was the most common mechanism of lower limb injury.

The incidence of abdominal injuries was $4.92 \%$ of cases and the most commonly involved vessels were the abdominal aorta and an inferior vena cava and all of these cases presented with hypovolemic shock at hospital admission and resulted in death. These results are in agreement with Hansen et al. ${ }^{20}$ who stated that, irrespective of whether the mechanism of injury was open or closed, abdominal injuries have a high risk of involving the inferior vena cava and abdominal aorta, in addition to injuries to several different organs, and have high mortality rates.

Vascular injuries can be classified into specific types that determine clinical status. In the majority of cases, vascular injuries can be hemorrhagic, ischemic or hemorrhagic/ischemic and can provoke compartment syndromes, and they can also present with hematoma, which is very frequent at hospital admission, presenting a clinical challenge to salvage of the limb and achieving hemodynamic stability. ${ }^{2,8}$ This study found that there was active hemorrhage in more than $50 \%$ of cases at hospital admission, followed in frequency by hematoma, ischemia and hypovolemic shock. Summed, the incidence rates of compartment syndrome and cardiac tamponade syndrome together affected less than $25 \%$ of the cases admitted during the period. Analysis of the data confirmed that there is an 
association between the structure injured and clinical presentation, by which the majority of cases of active hemorrhage and hematoma were the result of injuries to arteries, and also associations between description of vascular injury and the structure injured. As such, identification of these associations provides a basis for more effective intervention with trauma victims, both in terms of more rapid identification of injury severity and also as a guide to the best management approach for the treating team to take.

In this study, $71.21 \%$ of cases of vascular injury were associated with fractures. The high incidence of this combination is because of the proximity of bones to blood vessels within the limbs and, as such, fractures are generally associated with complex injuries. ${ }^{8}$ In cases of vascular injury to limbs with concomitant fractures, acute peripheral ischemia is the most serious complication, with risk of developments such as tissue necrosis and anaerobic metabolism of injured muscles, which lead to liver and kidney failure. ${ }^{8}$ Several experimental and clinical studies have described injuries to limbs with a view to the underlying bases for vascular repair and control of secondary hemorrhages, controlled by appropriate techniques to suture the injury. ${ }^{4,8,11}$

In all cases, vascular repair surgery was conducted, and the most prevalence procedures were ligature of veins and arteries, followed by vascular exploration; autologous grafts; amputations; revascularizations; fasciotomies; pseudoaneurysm repairs; sutures, and synthetic grafts. Ligature procedures had the highest rate of hospital discharge, but also had the highest mortality rate. This illustrates the complexity of caring for the victims of vascular traumas ${ }^{21,22}$ and the difficulties involved with identifying associations between type of surgery and patient prognosis. Amputations were probably performed to control damage, in critical cases, in which extended surgery times could involve a risk of death, as highlighted in studies undertaken with military trauma victims. ${ }^{23}$ Studies conducted to investigate the behavior of arterial grafts concluded that autologous venous vascular grafts remain alive because they have true endothelium fed by the bloodstream, and that the tunica muscularis undergoes hypertrophy and takes ownership of the artery. Concepts acquired treating vascular traumatisms always serve as a source for incorporation of new techniques ${ }^{22}$ and, to date, this method remains the ideal choice for vascular transplantation. Autologous vein grafts exhibit better results for initial limb salvage than synthetic grafts. ${ }^{23}$

Postoperative outcomes included more than $60 \%$ of cases free from complications and leading to discharge after improvement. This satisfactory result can be linked to advances in surgical techniques and the participation of rehabilitation professionals. These two points differentiate the present study from those conducted with military trauma victims, which reported high rates of morbidity and mortality. ${ }^{21,22,24-26}$ In $14.02 \%$ of cases, there were postoperative amputations due to limb ischemia and infections. There were infections in fewer than $10 \%$ of cases. Infection is the greatest challenge to vascular surgery and it is responsible for thrombosis and failure of sutures and can cause hemorrhages at arterial ligatures and subsequent gangrene ${ }^{1,8}$ In the present study, infection was only associated with a single case in which there was dehiscence of the surgical wound.

Compartment syndrome occurred in $0.76 \%$ of cases; the acute form of compartment syndrome is common in traumatisms that cause edema and hemorrhage, and can also be caused by extrinsic compression, such as bindings, tourniquets, plaster and postural pressure on the limb. ${ }^{8}$ Just $1.14 \%$ of patients suffered functional limitations and these were due to neurological injuries secondary to the traumatic vascular injury or were associated with severe traumatic injuries involving many different tissues. ${ }^{8,19}$

The most common length of hospital stay was less than 1 week, accounting for more than $55 \%$ cases, and admission to the ICU was only necessary in 44 cases. The most common length of ICU stay was also less than 1 week (72.73\%). Postoperative examination may reveal color changes at the extremities, at the most distal part of the limb, such as pallor and cyanosis, in addition to coldness. ${ }^{19,22}$ It is also necessary to check for other signs and symptoms, such as discrepancies between pulses, pale skin, coldness, paresthesia and restricted motor functions, since these symptoms are signs of nerve ischemia. ${ }^{19}$ An adequate length of hospital stay is therefore necessary to allow for assessment of these injuries and of the process of postoperative recovery. In Brazil, the number of urgent admissions to treat sequelae from external causes was 1,336 cases in 2011 across all age groups, increasing to 2,038 cases in 2013; and with this increase in demand hospitals need to draw on the specialties of a variety of professionals to collaborate together for the rehabilitation of sequelae caused by traumas in general. ${ }^{12,19}$ Physiotherapists are most often called upon, followed by psychologists, occupational therapists and speech and hearing specialist; however, in the majority of the cases studied here, patients did not receive care from any of these professionals. The importance of these health professionals to rehabilitation is recognized, intervening in a range of different ways to ensure patients recover more 
quickly and with better quality of life; however none of these professionals were involved in at least $40 \%$ of cases. To date, there are no studies in the literature describing these professionals' involvement exclusively in vascular trauma cases. As such, such an approach is an interesting avenue for future research.

The characteristics of the vascular traumas described in this study made possible an analysis of technical procedures, involving a wide range of several different areas of healthcare and administration of public resources. As such, the study contributes to enriching the theory on traumas and provides a basis for establishing prevention and control strategies, in view of the fact that traumas have become a public health problem.

\section{CONCLUSIONS}

Among this sample of trauma victims from the Brazilian state of Pará, patients were predominantly civilians, males, of working age and not from the state capital. Urban violence and traffic accidents were the primary causes of vascular traumas. Arterial vascular structures were most often injured and lower limbs were the most common anatomic site of injury. The participation of rehabilitation professionals in this case series was modest. Postoperatively, patients did not in general exhibit complications and the majority were discharged after exhibiting improvement, but the number of trauma victims who suffered amputation and/or died was significant.

\section{REFERENCES}

1. Senefonte FRA, Rosa GRPS, Comparin ML, et al. Amputação primária no trauma: perfil de um hospital da região centro-oeste do Brasil. J Vasc Bras. 2012;11(4):269-76. http://dx.doi.org/10.1590/ S1677-54492012000400004.

2. Costa-Val R, Campos-Christo SF, Abrantes WL, Campos-Christo MB, Marques MC, Miguel EV. Reflexões sobre o trauma cardiovascular civil a partir de um estudo prospectivo de 1000 casos atendidos em um centro de trauma de nível I. Rev Col Bras Cir. 2008;35(3):162-7. http://dx.doi.org/10.1590/S0100-69912008000300005.

3. Mattox KL, Feliciano DV, Burch J, Beall AC Jr, Jordan GL Jr, De Bakey ME. Five thousand seven hundred sixty cardiovascular injuries in 4459 patients. Epidemiologic evolution 1958 to 1987. Ann Surg. 1989;209(6):698-705, discussion 706-7. http://dx.doi. org/10.1097/00000658-198906000-00007. PMid:2730182

4. Antunes LF, Baptista A, Moreira J, et al. Traumatismos vasculares revisão de 5 anos. Angiologia e Cirurgia Vascular. 2011;7(2):86-93.

5. World Health Organization - WHO. World health statistics 2010. France: WHO; 2010.

6. Brasil. Ministério da Saúde. DATASUS/SI. Brasília: Ministérios da Saúde; 2014.

7. Moreira RCR. Trauma vascular.J Vasc Bras. 2008;7(2):185-6. http:// dx.doi.org/10.1590/S1677-54492008000200018.
8. Popescu Gl, Lupescu O, Nagea M, Patru C. Diagnosis and treatment of limb fractures associated with acute peripheral ischemia. Chirurgia (Bucur). 2013;108(5):700-5. PMid:24157116.

9. Magno LDP, Pereira AJF, Gonçalves BM, Almeida RMVR, Guimarães AGM, Bichara CNC. Escalpelamento nos Rios da Amazônia: um problema de saúde pública. Rev Para Med. 2012;26(1)

10. Lima-Costa MF, Barreto SM. Tipos de estudos epidemiológicos: conceitos básicos e aplicações na área do envelhecimento. Epidemiol Serv Saúde. 2003;12(4):189-201. http://dx.doi.org/10.5123/ S1679-49742003000400003.

11. Kauvar DS, Sarfati MR, Kraiss LW. National trauma databank analysis of mortality and limb loss in isolated lower extremity vascular trauma. J Vasc Surg. 2011;53(6):1598-603. http://dx.doi. org/10.1016/j.jvs.2011.01.056. PMid:21514772

12. Sohn VY, Arthurs ZM, Herbert GS, Beekley AC, Sebesta JA. Demographics, treatment, and early outcomes in penetrating vascular combat trauma. Arch Surg. 2008;143(8):783-7. http:// dx.doi.org/10.1001/archsurg.143.8.783. PMid:18711039

13. Lwanga SK, Lemeshow S. Sample size determination in health studies: a practical manual. Geneva: World Health Organization; 1991.

14. Costa CA, Baptista-Silva JCC, Rodrigues LME, Mendonça FLP, Paiva TS, Burihan E. Traumatismos de veia cava inferior. Rev Col Bras Cir. 2005;32:244-50.

15. Fatimi SH, Hanif HM, Awais A, Shamsi G, Muzaffar M. Major thoracic vessels and cardiac trauma: case series from a center in a developing country. Ulus Travma Acil Cerrahi Derg. 2012;18(6):4904. http://dx.doi.org/10.5505/tjtes.2012.39225. PMid:23588907

16. Reynolds RR, McDowell HA, Diethelm AG. The surgical treatment of arterial injuries in the civilian population. Ann Surg. 1979;189(6):700-8. http://dx.doi.org/10.1097/00000658-197906000-00005. PMid:453941

17. Markov NP, DuBose JJ, Scott D, et al. Anatomic distribution and mortality of arterial injury in the wars in Afghanistan and Iraq with comparison to a civilian benchmark. J Vasc Surg. 2012;56(3):72836. http://dx.doi.org/10.1016/j.jvs.2012.02.048. PMid:22795520

18. Mendieta-Azcona C, Gandarias-Zúñiga C, Ocaña-Guaita J, et al. Traumatismo por arma de fuego en la arteria femoral superficial. Angiologia. 2004;56(1):67-74. http://dx.doi.org/10.1016/ S0003-3170(04)74850-6.

19. Silva MAM, Burihan MC, Barros OC, et al. Trauma vascular na população pediátrica. J Vasc Bras. 2012;11(3):199-205. http:// dx.doi.org/10.1590/S1677-54492012000300006.

20. Hansen C), Bernadas C, West MA, et al. Abdominal vena caval injuries: outcomes remain dismal. Surgery. 2000;128(4):572-8. http://dx.doi.org/10.1067/msy.2000.108054. PMid:11015090

21. Fingerhut $A$, Leppäniemi AK, Androulakis GA, et al. The European experience with vascular injuries. Surg Clin North Am. 2002;82(1):175-88. http://dx.doi.org/10.1016/S0039-6109(03)001476. PMid:11905944

22. Bowley DMG, Degiannis E, Goosen J, Boffard KD. Penetrating vascular trauma in Johannesburg, South Africa. Surg Clin North Am. 2002;82(1):221-35. http://dx.doi.org/10.1016/S0039-6109(03)001518. PMid:11908509

23. Brown KV, Ramasamy A, Tai N, MacLeod J, Midwinter M, Clasper JC. Complications of extremity vascular injuries in conflict. J Trauma. 2009;66(4, Suppl):S145-9. http://dx.doi.org/10.1097/ TA.0b013e31819cdd82. PMid:19359958

24. Fox CJ, Gillespie DL, Cox ED, et al. Damage control resuscitation for vascular surgery in a combat support hospital. J Trauma. 2008a;65(1):1-9. http://dx.doi.org/10.1097/TA.0b013e318176c533. PMid:18580508 
25. Fox CJ, Gillespie DL, Cox ED, et al. The effectiveness of a damage control resuscitation strategy for vascular injury in a combat support hospital: results of a case control study. J Trauma. 2008b;64(2, Suppl):S99-106, discussion S106-7. http://dx.doi.org/10.1097/ TA.0b013e3181608c4a. PMid:18376180

26. Fox CJ, Perkins JG, Kragh JF Jr, Singh NN, Patel B, Ficke JR. Popliteal artery repair in massively transfused military trauma casualties: a pursuit to save life and limb. J Trauma. 2010;69(1, Suppl 1):S123-34. http://dx.doi.org/10.1097/TA.0b013e3181e44e6d. PMid:20622606
Correspondence Ludmylla Teixeira Soares

Universidade do Estado do Pará, Hospital Metropolitano de Urgência e Emergência

Travessa do Chaco, 1320 - Marco CEP 66085-451 - Belém (PA), Brazil Tel: +55 (91) 98154.1574 E-mail: ludmylla_19@hotmail.com

Author information

LTS - Resident physician in Trauma Urgency and Emergency, Hospital Metropolitano de Urgência e Emergência (HMUE) of Pará State. CCB - Physical therapist and preceptor of the Multiprofessional

Residence Program, Hospital Metropolitano de Urgência e Emergência (HMUE) of Pará State. AKJ - Vascular and endovascular surgeon and interventional radiologist, Hospital Metropolitano de Urgência e Emergência (HMUE) of Pará State. AJFP - MSc and PhD candidate in Biomedical Engineering from Universidade Federal do Rio de Janeiro (UFRJ).

Author contributions Conception and design: LTS, CCB, AKJ, AJFP Analysis and interpretation: LTS, CCB, AKJ, AJFP Data collection: LTS, AKJ Writing the article: LTS, CCB, AJFP Critical revision of the article: $L T S, C C B, A K J$, AJFP Final approval of the article*: LTS, CCB, AKJ, AJFP Statistical analysis: AJFP Overall responsibility: LTS

*All authors have read and approved of the final version of the article submitted to J Vasc Bras. 